\title{
Delta undulator for Cornell energy recovery linac
}

\author{
Alexander B. Temnykh* \\ Cornell University, LEPP, Ithaca, New York 14853-8001, USA
}

(Received 17 October 2008; published 11 December 2008)

\begin{abstract}
In anticipation of a new era of synchrotron radiation sources based on energy recovery linac techniques, we designed, built, and tested a short undulator magnet prototype whose features make optimum use of the unique conditions expected in these facilities. The prototype has pure permanent magnet (PPM) structure with $24 \mathrm{~mm}$ period, $5 \mathrm{~mm}$ diameter round gap, and is $30 \mathrm{~cm}$ long. In comparison with conventional undulator magnets it has the following: (i) full $x$-ray polarization control.- It may generate varying linear polarized as well as left and right circular polarized x rays with photon flux much higher than existing Apple-II-type devices. (ii) 40\% stronger magnetic field in linear and approximately 2 times stronger in circular polarization modes. This advantage translates into higher x-ray flux. (iii) Compactness.-The prototype can be enclosed in a $\sim 20 \mathrm{~cm}$ diameter cylindrical vacuum vessel. These advantages were achieved through a number of unconventional approaches. Among them is control of the magnetic field strength via longitudinal motion of the magnet arrays. The moving mechanism is also used for $\mathrm{x}$-ray polarization control. The compactness is achieved using a recently developed permanent magnet soldering technique for fastening PM blocks. We call this device a "Delta" undulator after the shape of its PM blocks. The presented article describes the design study, various aspects of the construction, and presents some test results.
\end{abstract}

DOI: 10.1103/PhysRevSTAB.11.120702

PACS numbers: 85.70.- w, 41.60.- m, 07.85.Qe

\section{INTRODUCTION}

Synchrotron radiation facilities based on energy recovery linacs (ERL) will have a number of specific features, which could be exploited for superior insertion device design. In comparison with storage rings, they will have smaller horizontal beam emittance and consequently smaller horizontal beam size. In addition, they will not require extra beam aperture to provide space for residual oscillation of the injected particles. Furthermore, ERL facilities can tolerate a much bigger total one-pass beam loss than storage rings that require adequate beam lifetime. All this makes possible a reduction of horizontal beam aperture to the size of the vertical, i.e., the beam aperture can be round. Round gap (bore) insertion devices, in turn, may significantly facilitate the design as well as enhance magnetic field properties. Following this argument we chose a $5 \mathrm{~mm}$ diameter round bore. The roundness provides symmetry of the design and a $5 \mathrm{~mm}$ diameter seemed to be large enough for access for magnetic field measurements.

Field strength is controlled by an adjustable phase (AP) scheme. In this scheme the peak field is controlled by moving the magnetic arrays relative to each other in the longitudinal direction. The AP scheme is compact and does not require a large external frame to hold magnet arrays and for gap control. Moreover, the mechanism providing

\footnotetext{
*Current address: A. Temnykh, Wilson Lab, Cornell University, NY 14850, USA. abt6@cornell.edu
}

magnetic array longitudinal motion can be used for x-ray polarization and photon spectrum control. The AP scheme's theoretical model has been developed by Roger Carr in Refs. [1,2]. Electron beam test results were described in [3]. Presently, one AP-type undulator successfully operates as an X-ray source for the "ADRESS" beam line at Swiss Light Source. This undulator provides $\mathrm{x}$ rays in the energy range between 400 and $1800 \mathrm{eV}$ with circular and variable linear polarization: see the website [4].

The round bore allows the design of a highly symmetric magnetic structure. On the one side, magnetic structure can be considered as a combination of two identical undulators rotated by $90^{\circ}$ relative to each other around the beam axis. On the other, it can also be considered as a kind of AppleIII structure mentioned in Ref. [5]. The Delta structure has similarities with the undulators described in [6,7] as well. The structure can provide $\sqrt{2}$ times stronger planar magnetic field than conventional planar pure permanent magnet (PPM) undulators and approximately 2 times stronger helical field if compared with existing Apple-II-type undulators of the same gap to period ratio.

The newly developed PM magnet block fastening technique is the key to the design compactness. Mechanical fastening methods were considered as not very practical, because they would take additional space and, probably, would require more complex magnetic block shape. The latter could considerably increase the cost and the realization time of the project. Gluing was also undesirable because it would not be compatible with in-vacuum operation. After appropriate investigation we developed a technique for soldiering of $\mathrm{Ni}$ coated $\mathrm{NdFeB}(40 \mathrm{SH})$ magnets 
to copper holders without demagnetization [8]. This technique was used in the construction.

It should be mentioned that the conceptual idea for such a PPM undulator was presented in 2006 [9]. Encouraged by interest from the Cornell x-ray users' community, this idea evolved into a detailed design and recently a prototype was built and tested.

\section{MODEL DESCRIPTION}

\section{A. General information}

A picture of the Delta undulator prototype is shown on Fig. 1 . The prototype is $\sim 30 \mathrm{~cm}$ long, $\sim 15 \mathrm{~cm}$ high, and $\sim 15 \mathrm{~cm}$ wide. The magnetic structure consists of two pairs of magnetic arrays as depicted in the computer generated Fig. 2 with major components numbered. One pair provides vertical field and another horizontal. Magnet arrays (1) are assembled on baseplates (2). To provide longitudi-

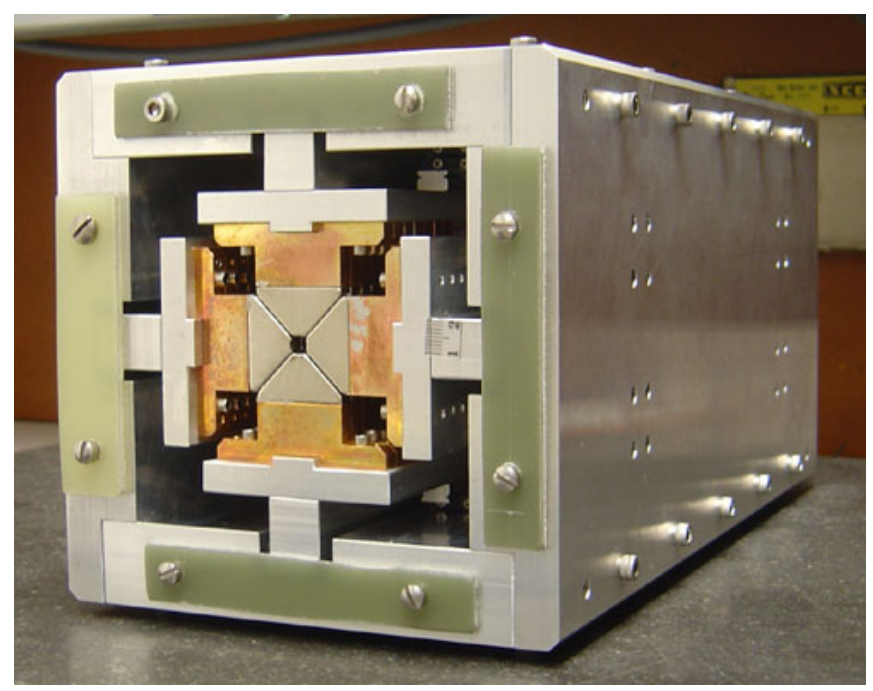

FIG. 1. (Color) Delta undulator prototype view.
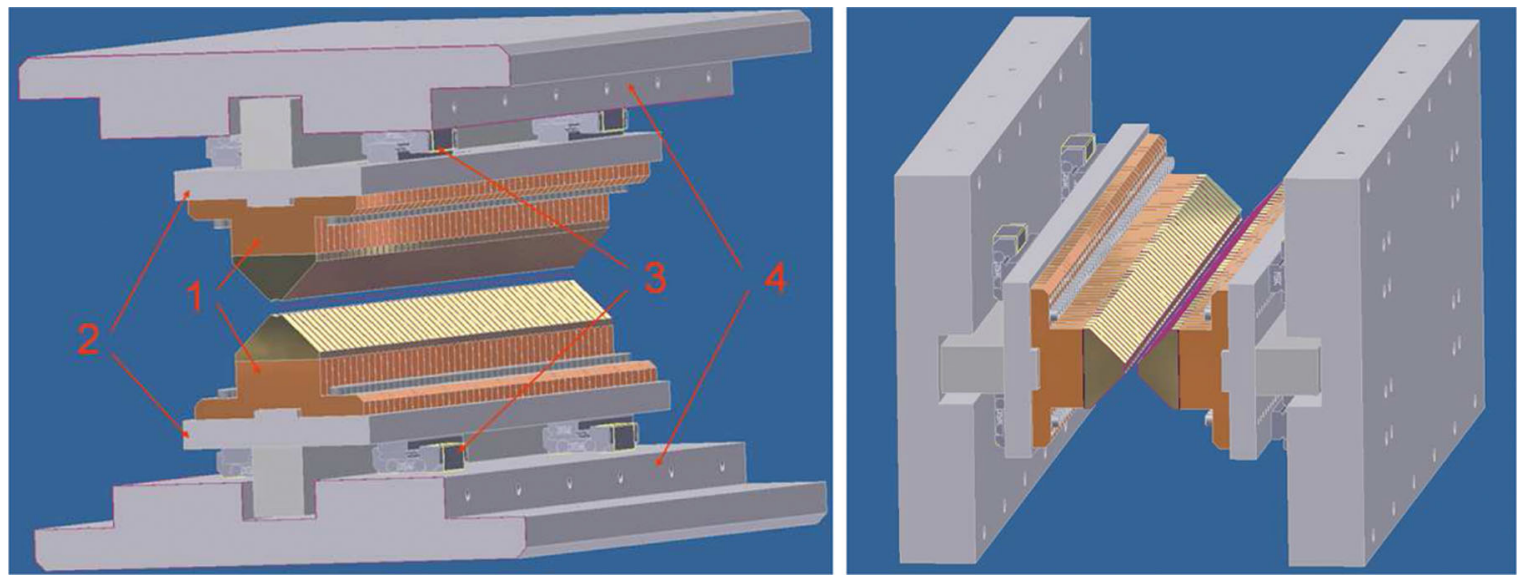

FIG. 2. (Color) Computer generated view. Left plot—magnetic arrays providing vertical field. Here (1)—magnetic arrays, (2)— baseplates, (3)—rails providing longitudinal motion, (4)—plates forming the rigid frame. Right plot—arrays providing horizontal field. 


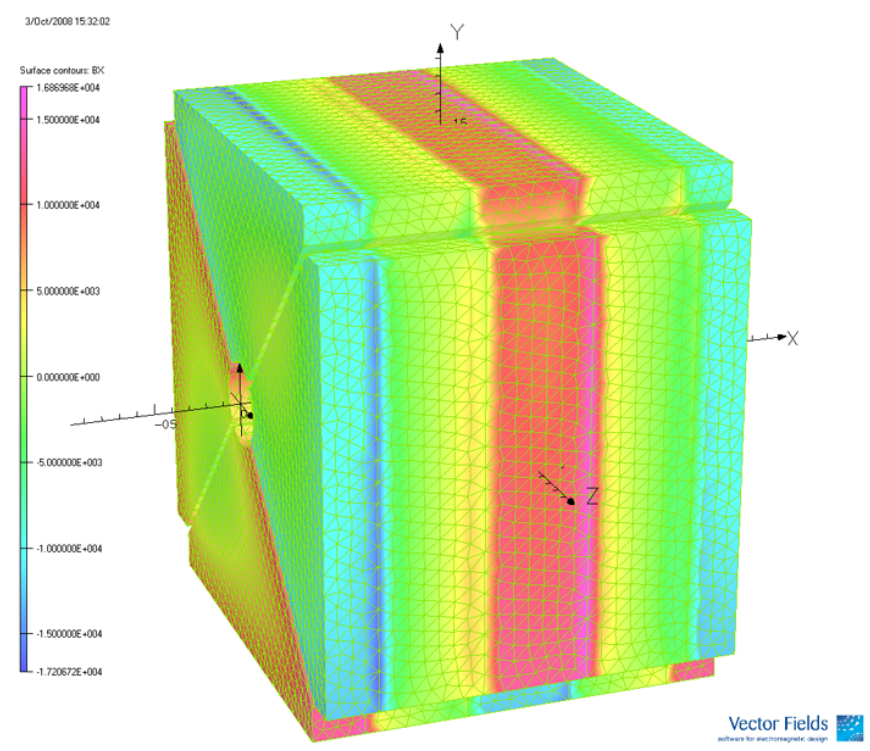

FIG. 3. (Color) 3D model of one period of Delta undulator magnetic structure used in field calculation.

longitudinal coordinate (left) and field variation across the bore (right). The left plot indicates $\simeq 1 \mathrm{~T}$ peak field and $90^{\circ}$ phase shift between vertical and horizontal components. The field variation $d B / B$ across the bore, see right plot, in the $\pm 1 \mathrm{~mm}$ region around the axis can be approximated as $d B / B \simeq-0.02 \times d^{2}$, where $d$ is a distance from axes in $\mathrm{mm}$.

Figure 5 depicts magnetic field characteristics in the planar mode. In this mode the vertical and horizontal field components are in phase. The left plot shows two orthogonal field components $\left(B_{y}+B_{z}\right) / \sqrt{2}$ and $\left(B_{y}-B_{z}\right) / \sqrt{2}$ on

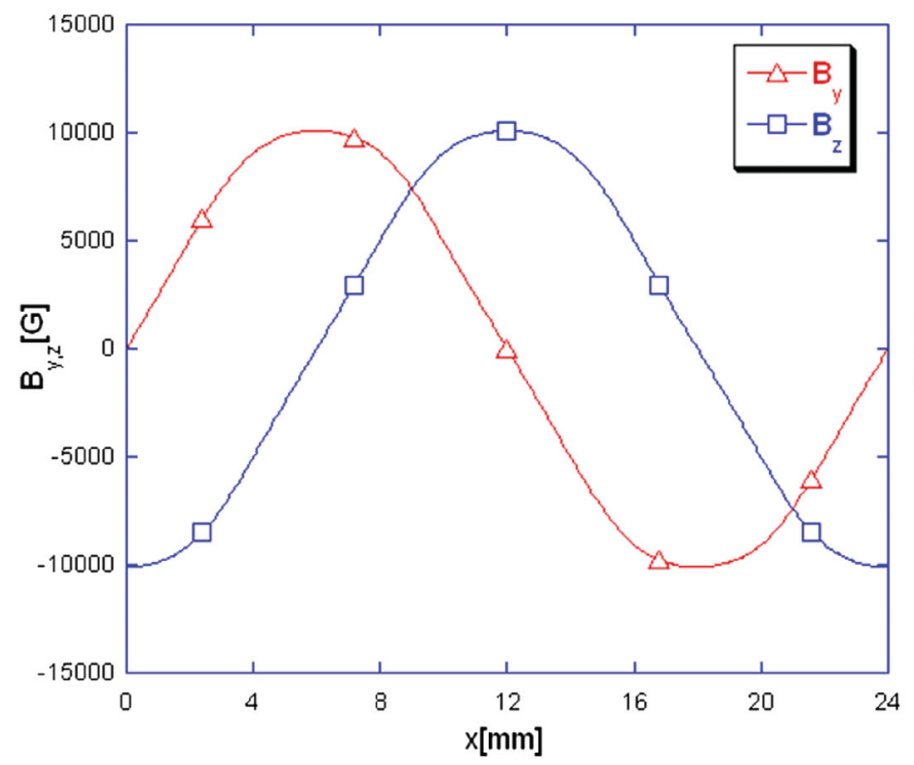

beam axis as a function of longitudinal coordinate. The first component has maximum $\sim 1.43 \mathrm{~T}$, the second is "zero." The field variation in the $\pm 1 \mathrm{~mm}$ region around the beam axis can be described as $d B / B \simeq-0.0037 \times d^{2}$, where $d$ is a distance from axes in $\mathrm{mm}$.

To provide data for the possible optimization, we calculated dependence of the peak field on period for both helical and planar modes. The data plotted on Fig. 6 (left) was fitted with commonly used approximation,

$$
B[T]=a \exp \left[b \frac{g}{\lambda_{0}}+c\left(\frac{g}{\lambda_{0}}\right)^{2}\right]
$$

where $\lambda_{0}$ is the period and $g$ is the gap (see Refs. [5,10]). The fit yielded coefficients depicted in Table I.

Plots on Fig. 6 (right) compare the calculated peak field for the Delta type of structure as a function of gap over period ratio with others. Data for "PPM planar vertical/ horizontal field" and "PPM helical field" were found in Ref. [10], characteristics for Apple-III structure are from [5]. This comparison evidently shows advantage of the Delta-type structure.

\section{Mechanical considerations}

Dimensions of main elements such as baseplates and plates forming the rigid frame where chosen after extensive stress analysis with the programs INVENTOR [11] and ANSYS [12]. Magnetic field calculations indicated that magnetic forces will be applied to PM blocks mostly in the region adjacent to the beam axis. In helical mode, the forces will be directed toward the beam axis and will be approximately $45 N$ per structure period. This will cause the baseplate deformation shown in Fig. 7(left) with $2 \mu \mathrm{m}$

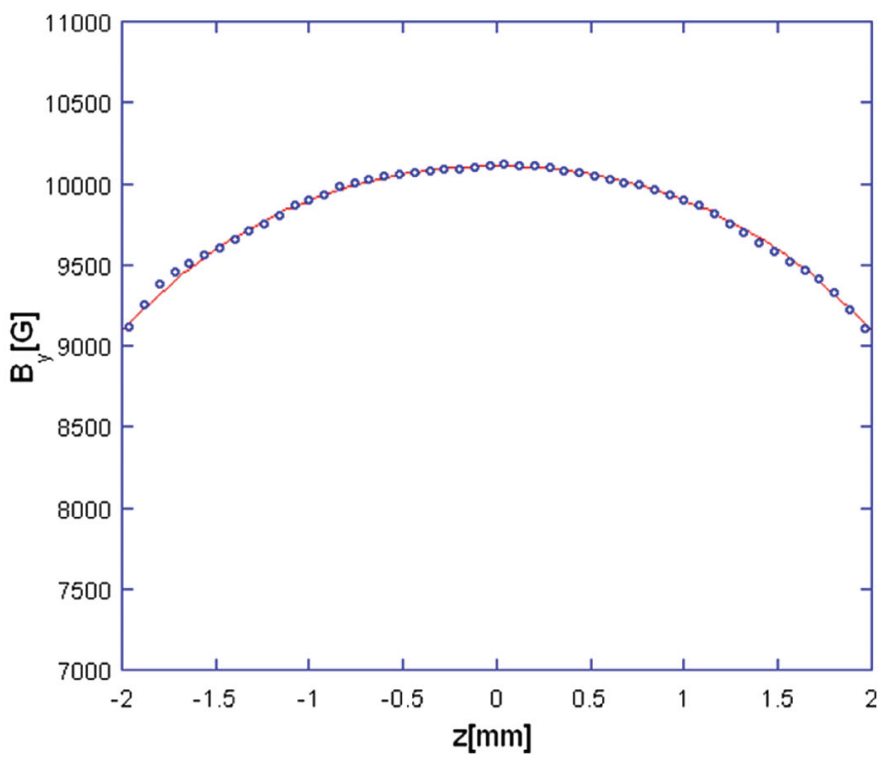

FIG. 4. (Color) Helical mode. Left plot—vertical and horizontal field components along the beam axis, $B_{\max }=1.01 \mathrm{~T}$. Right plotfield variation across the bore. In the $\pm 1 \mathrm{~mm}$ region field variation $d B / B \simeq-0.020 \times d^{2}$, where $d$ is a distance from the beam axes in $\mathrm{mm}$. 

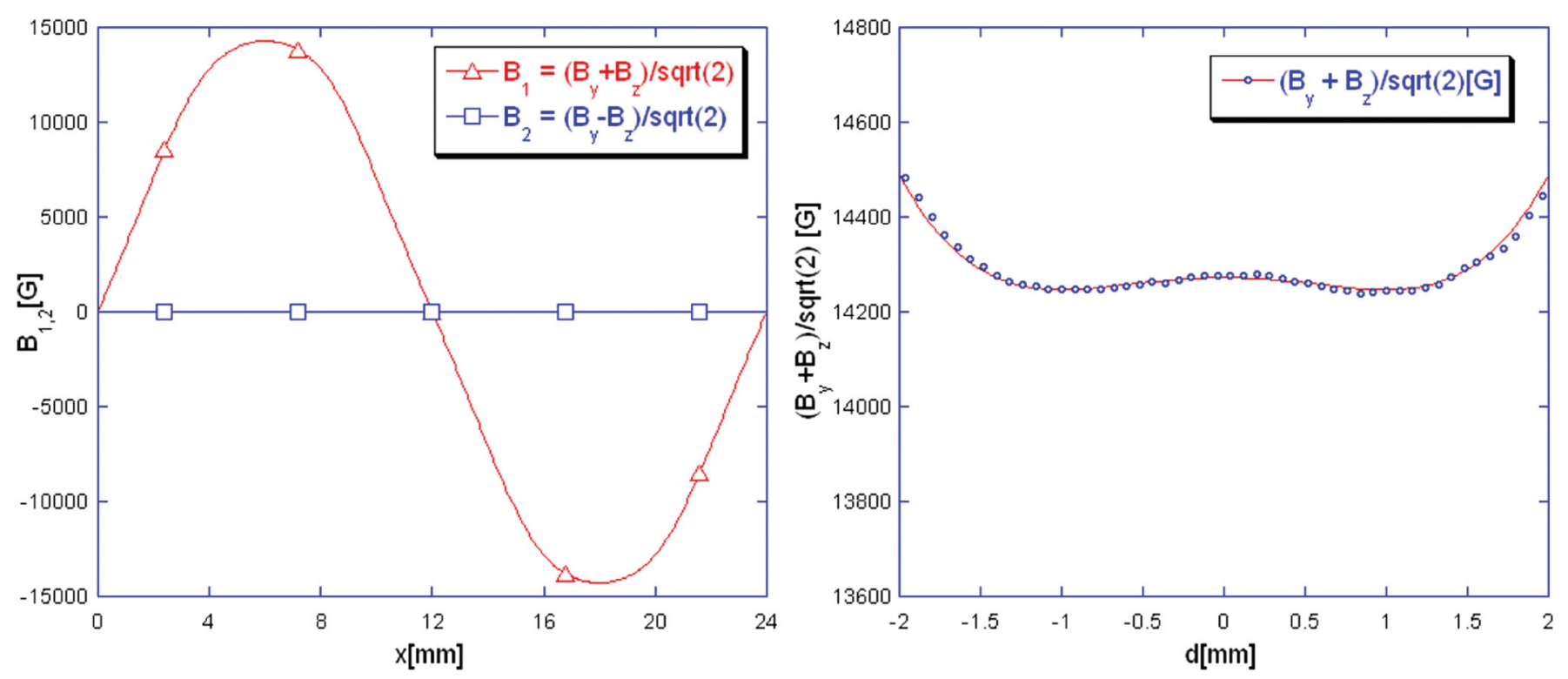

FIG. 5. (Color) Planar mode. Left plot-two orthogonal field components versus the coordinate along the beam axis, $B_{\text {max }} \simeq 1.43$ T. Right plot-field variation across the bore. In the $\pm 1 \mathrm{~mm}$ region the field variation $d B / B \simeq-0.0037 \times d^{2}$, where $d$ is the distance from the beam axis in mm.

TABLE I. Peak field fit parameters for Delta undulator.

\begin{tabular}{lccc}
\hline \hline Mode & $a$ & $b$ & $c$ \\
\hline Planar field & 1.96 & -0.82 & -3.31 \\
Helical field & 1.45 & -1.28 & -2.24 \\
\hline \hline
\end{tabular}

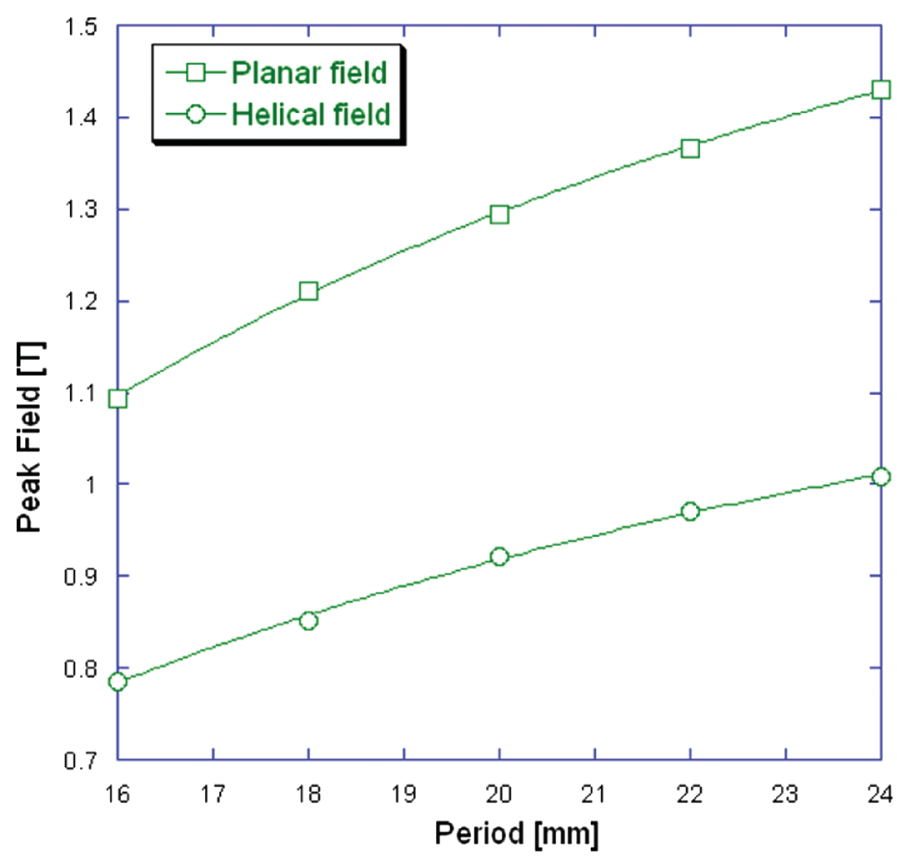

maximum. In planar mode magnetic forces will be quite different. In addition to the $45 \mathrm{~N}$ directed to the beam axis, there will be an additional $282 \mathrm{~N}$ perpendicular to the axis due to repulsing and attracting from the adjoint arrays. The latter will produce $659 \mathrm{~N}-\mathrm{cm}$ torque. The resultant deformation will have a profile shown on Fig. 7(right) with

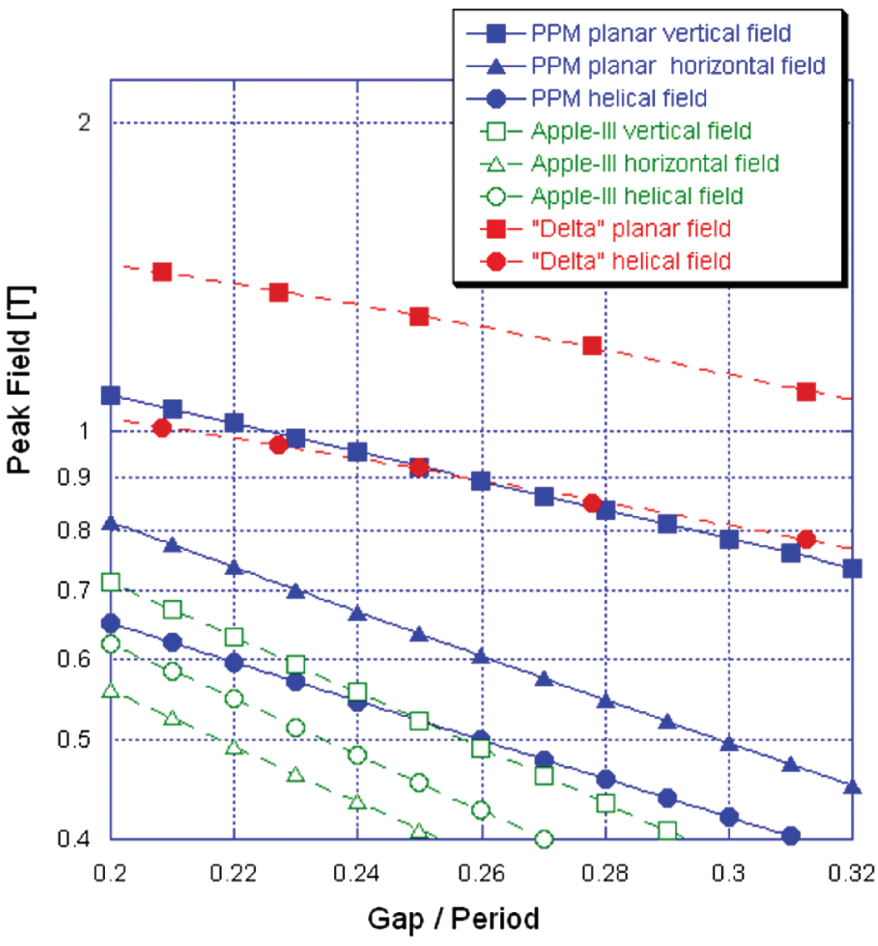

FIG. 6. (Color) Left plot-peak field in planar and helical mode as a function of period for the 5 mm diameter bore and magnetic material with $B_{r}=1.26 \mathrm{~T}$ consisting of $\mathrm{NdFeB}(40 \mathrm{SH})$ specification. Right plot—peak field versus gap over the period ratio for Delta and other magnetic structures. 

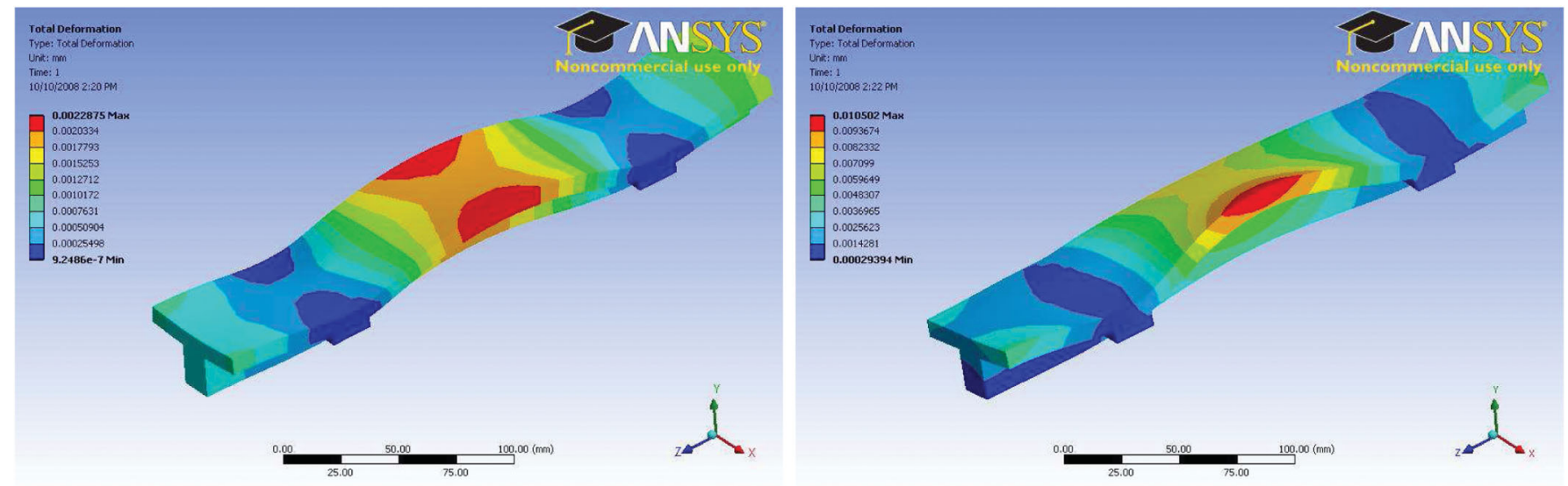

FIG. 7. (Color) Baseplate deformation in helical (left plot) and planar field (right plot) modes.

$10 \mu \mathrm{m}$ maximum. This is larger than in the case of the helical mode, but still acceptable. Note that baseplate deformations were minimized by optimizing the mounting rail locations.

This analysis also indicated that in the helical mode mounting rails will be stressed by $116 \mathrm{~N}$ and in the planar by $800 \mathrm{~N}$ forces. In the prototype we used mounting rails LU09AR from "NSK NIPPON SEIKO" rated up to $1700 \mathrm{~N}$ of static load.

The rigid frame deformation calculated for the helical and planar modes is given in Fig. 8. In the case of the helical field the maximum frame deformation will be less than $1 \mu \mathrm{m}$ and can be neglected. In the planar mode the frame deformation will be much larger, $\sim 20 \mu \mathrm{m}$. However, because it is uniform along the beam axis it will not cause variation of the field and undulator " $K$ " parameter which could degrade the x-ray spectrum.

\section{Beam heat load analysis}

In "Ultrafast" mode (extreme case) the Cornell ERL will operate with $1 \mathrm{nC}$ per bunch charge, $1 \mathrm{MHz}$ repetition

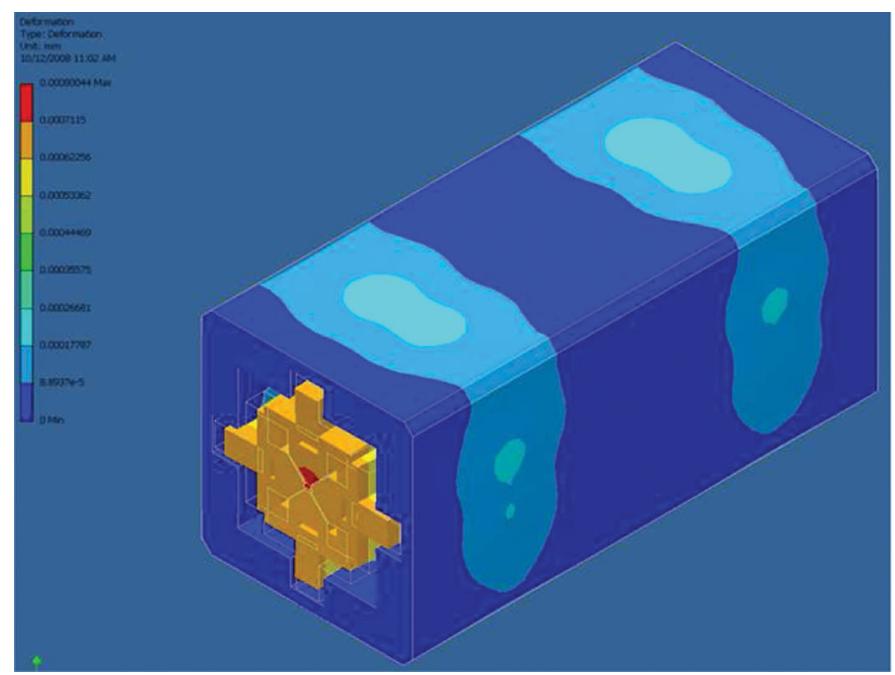

rate, and 0.1 psec bunch duration. Following Ref. [13] we estimated that in this mode the heat load generated by the beam image current in the walls of $5 \mathrm{~mm}$ diameter bore will be $28 \mathrm{~W} / \mathrm{m}$. For wall conductance we assume $6 \times$ $10^{7}[1 / \Omega / \mathrm{m}]$ for Ni coated thin copper tape placed on the top of PM blocks.

To provide a heat sink, two cooling elements will be attached to both sides of the baseplate as shown on Fig. 9. Using software ANSYS we found that under these conditions the maximum temperature rise will occur at the top of the magnet array and will not exceed $0.2^{\circ} \mathrm{C}$, see Fig. 9, which is acceptable.

\section{E. Vacuum on beam axis}

As an in-vacuum device, the vacuum performance of the Delta undulator magnet must be evaluated to ensure compatibility with the ultrahigh vacuum (UHV) requirement of the accelerator. To reduce damage from the synchrotron radiation, proper synchrotron radiation (SR) masking at the undulator entrance will prevent the direct SR strike of the undulator bore. Thus, there is no beam induced dynamic

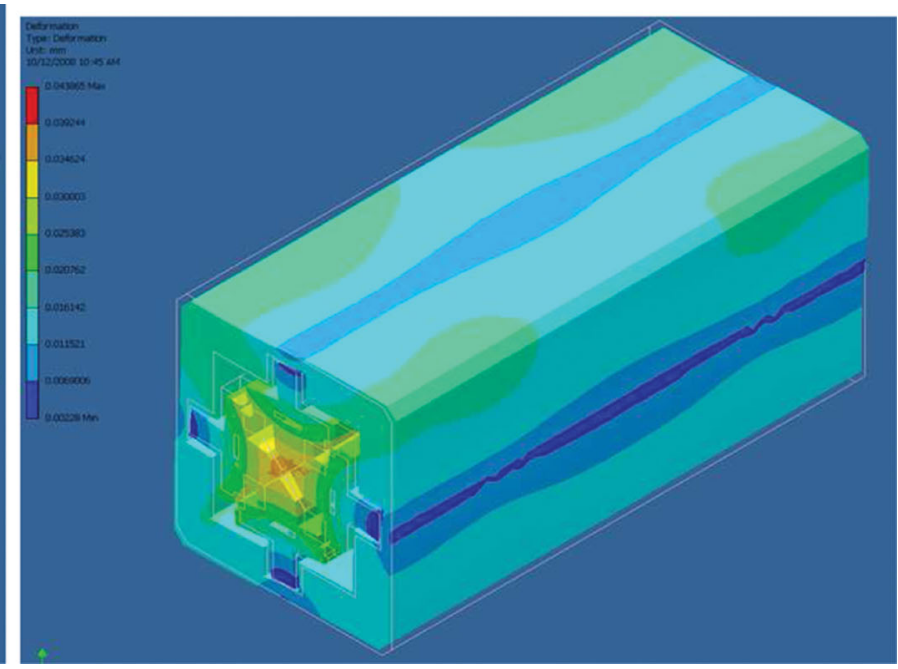

FIG. 8. (Color) Frame deformation for helical (left plot) and planar (right plot) modes of operation. 


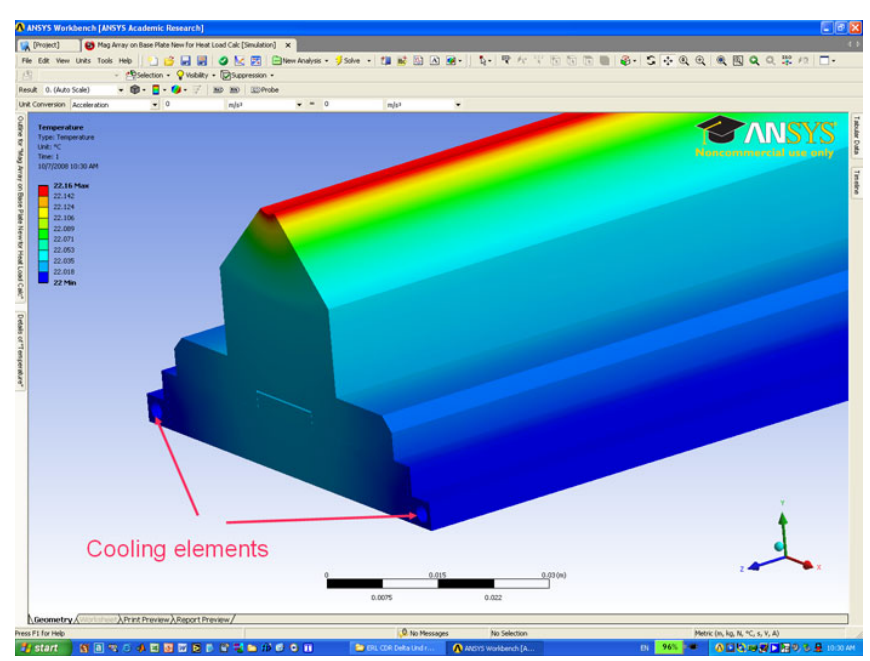

FIG. 9. (Color) Heat load analysis.

gas load in the magnet bore. One need only be concerned with the static residual pressure due to the thermal outgassing from the magnet pole materials. Tests [14] showed that with proper cleaning and degassing (through preassembling bakeout), the thermal outgassing rate of the $\mathrm{Ni}$ plated PM blocks can be reduced to below $4 \times$ $10^{-12}$ Torr-liter/ $\mathrm{sec} / \mathrm{cm}^{2}$, comparable with commonly used UHV materials such as stainless steel and copper. To prevent pressure buildup along the very small magnet bore, four $0.5-\mathrm{mm}$ wide venting gaps are designed into the magnet structure to connect the bore to the outer vacuum space, where adequate vacuum pumping is installed. Simple gas transport calculation (using the gas conductance formula given in [15]) shows that these venting gaps are sufficient to keep pressure in the magnet bore below $10^{-9}$ Torr. For the actual insertion device (with much longer magnet length), more careful pressure calculations are planned, using 3D Monte Carlo code (such as MOLFLOW [16]).

\section{SOME ASPECTS OF THE PROTOTYPE CONSTRUCTION AND TEST RESULTS}

PM blocks used for the Delta undulator prototype were ordered from "Stanford Material Corp." They are Delta like in shape and made of $\mathrm{NbFeB} 40 \mathrm{SH}$ material coated with $\sim 12 \mu \mathrm{m}$ of $\mathrm{Ni}-\mathrm{Cu}-\mathrm{Ni}$. All block dimensions and detailed material specifications are given on Fig. 10. The blocks were fastened to copper holders by a recently developed soldering method described in [8]. As solder we used $63 / 37 \mathrm{Sn} / \mathrm{Pb}$ alloy with rosin component and with a melting point of $183^{\circ} \mathrm{C}$. The given alloy has a tensile strength $54 \mathrm{MPa}$ which is much higher than for solders with lower melting temperature. To prevent PM demagnetization during the temperature rise needed for solder melting, the blocks were surrounded by steel plates. The steel redistributed the magnetic field in such a way that the demagnetizing field was reduced. This allowed raising the temperature above the solder melting point without risk of PM demagnetization. More details can be found in [8].

In the construction process PM blocks together with copper holders were enclosed in "steel jackets." The solder wire was flattened and inserted between them. Then the whole assembly was placed in the oven for 2 hours at $195^{\circ} \mathrm{C}$. With three sets of the soldering fixture, one person soldered three blocks in 3 hours.

A single Delta shape PM block and the block soldered to the copper holder are shown on Fig. 11. Note the holder has provision for mechanical fastening to the baseplate and for vertical position control.

\section{A. Magnetic field tuning results}

The magnetic field of each of the four magnet arrays has been measured with a Hall probe and tuned. The measurement setup consisted of a linear sliding stage, temperature compensated Hall probe, "LakeShore 455 DSP Gaussmeter" in connection with "HP3456A Digital Voltmeter" and stepping motor controller. For measurement control we used laptop "IBM ThinkPad" with LABVIEW. The field was measured "on the fly" with $0.25 \mathrm{~mm}$ step. For field analysis we used "B2E V3.3" software developed by the ESRF ID group. Because of small size and weight, the magnetic array was mounted on the moving stage while the Hall probe position was fixed. This configuration provided more stable measurement. Prior to the field measurement the setup was very carefully aligned.

Figure 12 gives the setup view. Here one can see the magnet array consisted of Delta shaped PM blocks. The array situated on the moving linear stage while the Hall probe is fixed. At the end of array there are two vertically displaced PM blocks. This displacement has been made for "end termination" purpose.

In the process of the field tuning we first measured the field, then analyzed it with "B2E" software. In the analysis we used the doubled field strength assuming an identical array placed symmetrically relative to the Hall probe above the tested. Trajectory corrections were made by displacing the vertically magnetized blocks; the phase was corrected by the blocks magnetized along the beam axis. These steps, measurement and correction, were repeated until trajectory and phases converged to a satisfactory level. Usually this occurred after 10-15 iterations.

Figure 13 illustrates the magnet array " 1 " tuning result. The first plot shows the measured field. The two others are trajectory and phase errors calculated for the doubled strength field. Both indicate satisfactory results. After the field tuning, three other arrays " 2 ", " 3 ", and "4" had similar trajectory and phase errors. 


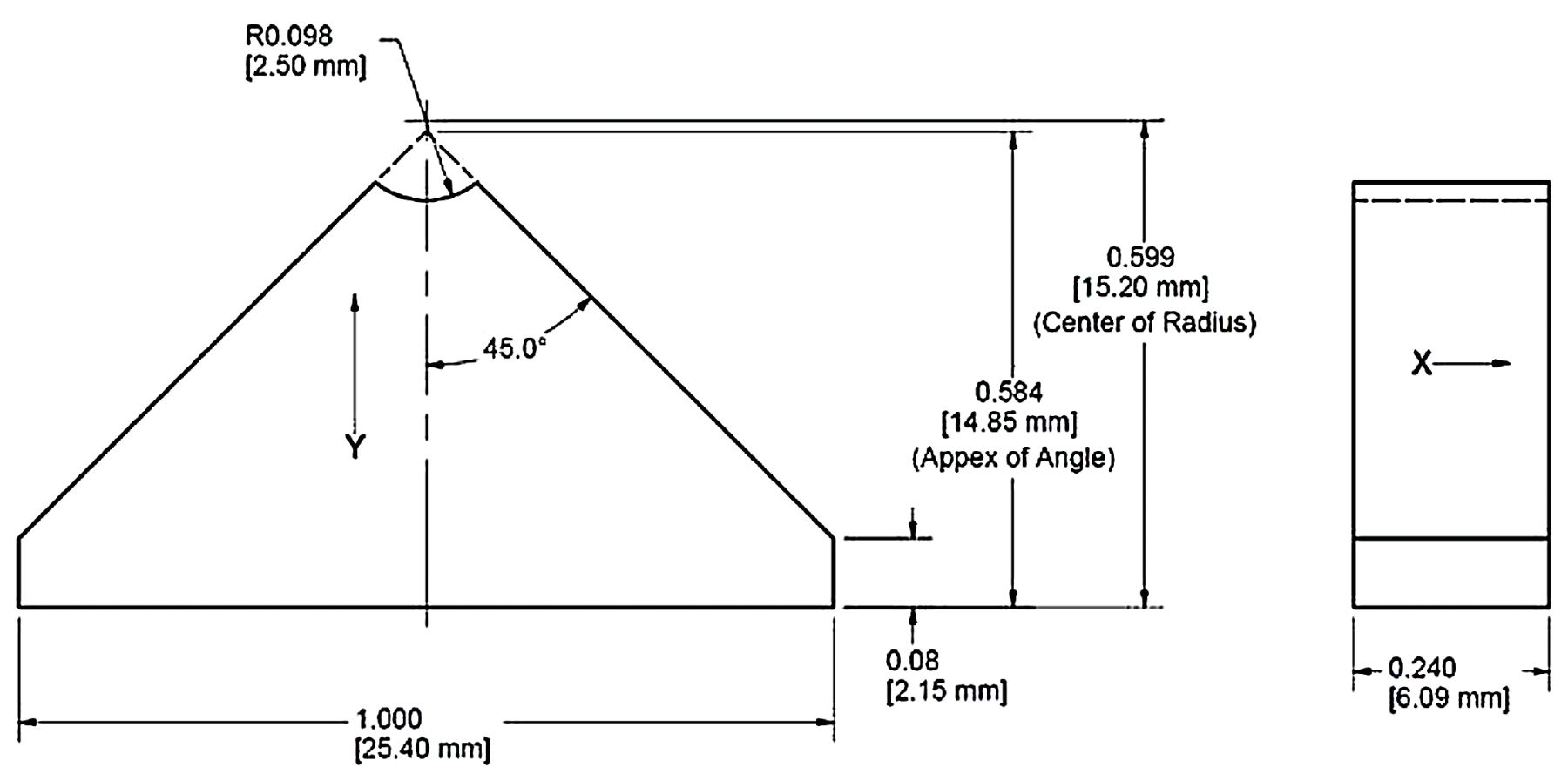

Demagnetization curves at different temperatures N40SH

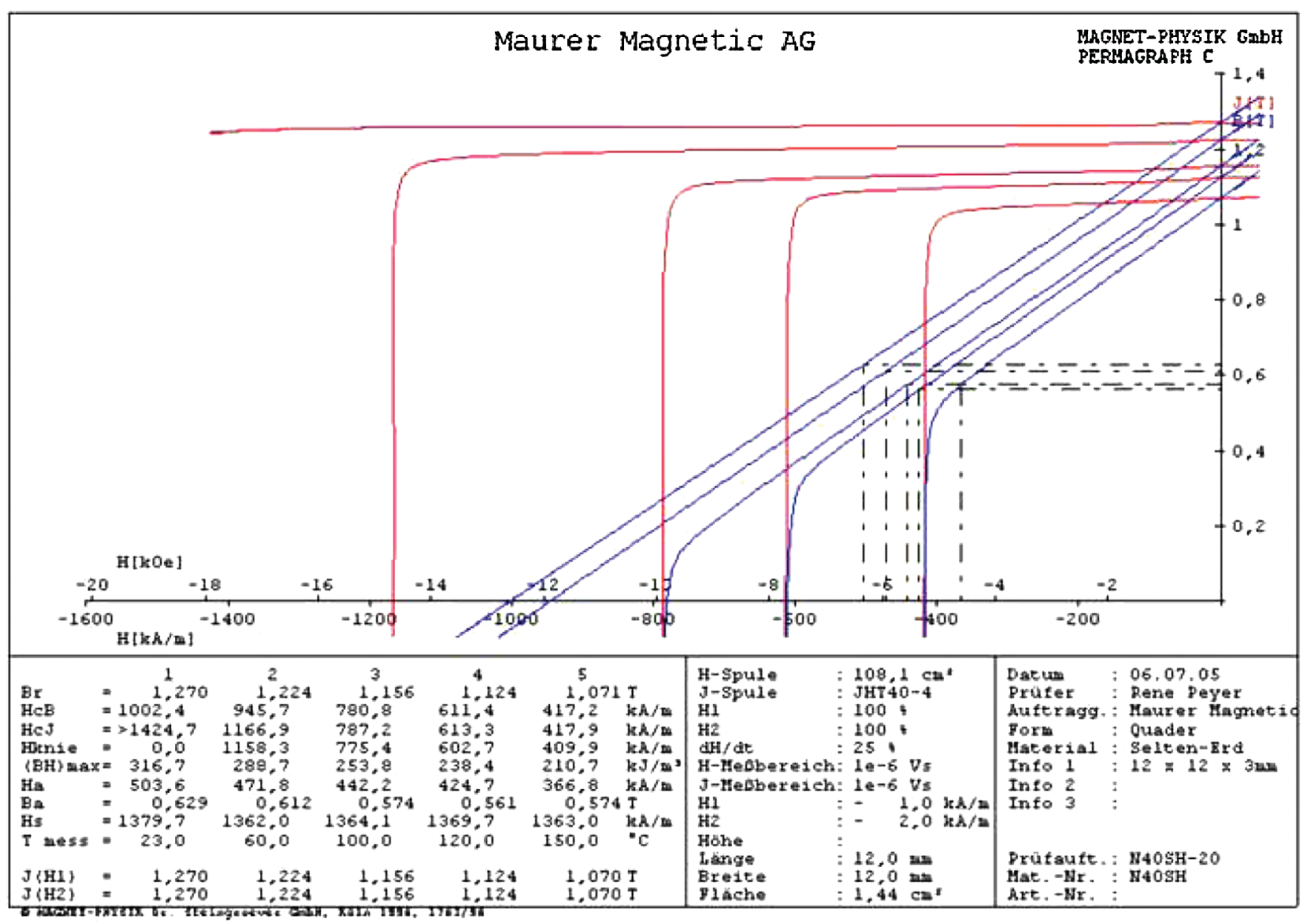

FIG. 10. (Color) PM dimensions and material specification. Material specification data was copied from the "Maurer Magnetic AG" web site. 

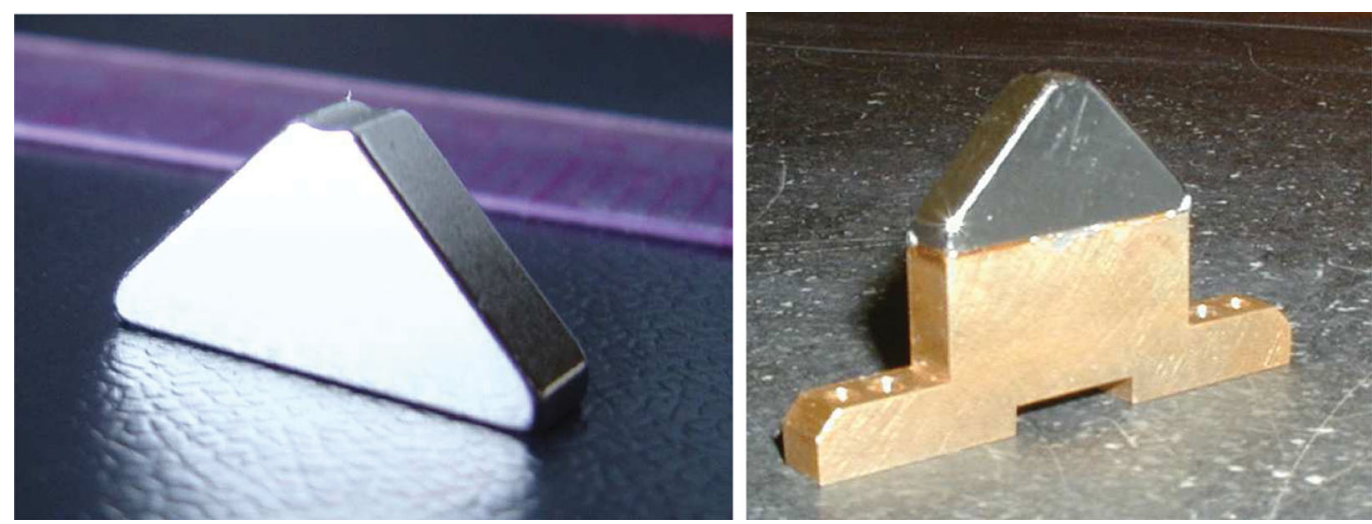

FIG. 11. (Color) Single Delta shape magnetic block (left), the block soldered to copper holder (right).

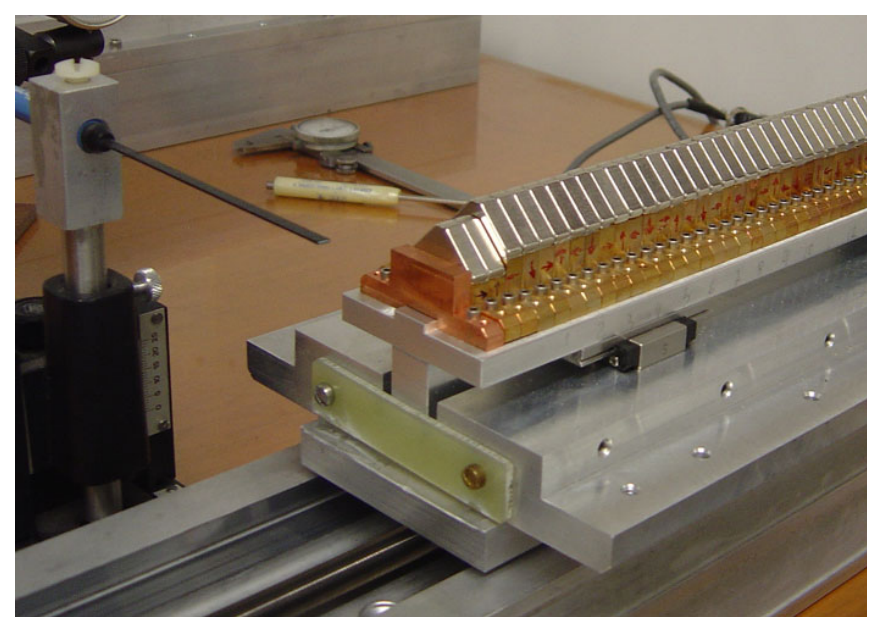

FIG. 12. (Color) Assembled array on the field measurement bench.

\section{B. Composite field analysis}

Because of linearity of the PPM structure, we can obtain the properties of the assembled undulator by combining individual array fields. Plots on Fig. 14 show this field for helical (left plot) and linear (right plot) modes of operation. One can notice that the peak field for both modes is in good agreement with the predicted 1 and $1.4 \mathrm{~T}$ field, respectively, see Sec. II B. In linear mode rms phase errors of the composed field were found to be $\sim 2.2^{\circ}$. This proves our method for the individual arrays field tuning procedure.

The high field quality was also confirmed by calculation of the photon flux spectrum with program SPECTRA [17]. Using field distributions combined from the measurements described above and corresponding to helical and planar modes, see Fig. 14, we calculated the spectrum of the photon flux coming through a $1 \mathrm{~mm}$ diameter slit at $50 \mathrm{~m}$ from the source generated by electron beam with $25 \mathrm{~mA}$ current, $8 \times 10^{-12} \mathrm{~m}$ rad emittance and $0.0002 \sigma_{e} / E_{0}$ energy spread. Those beam parameters are consistent with
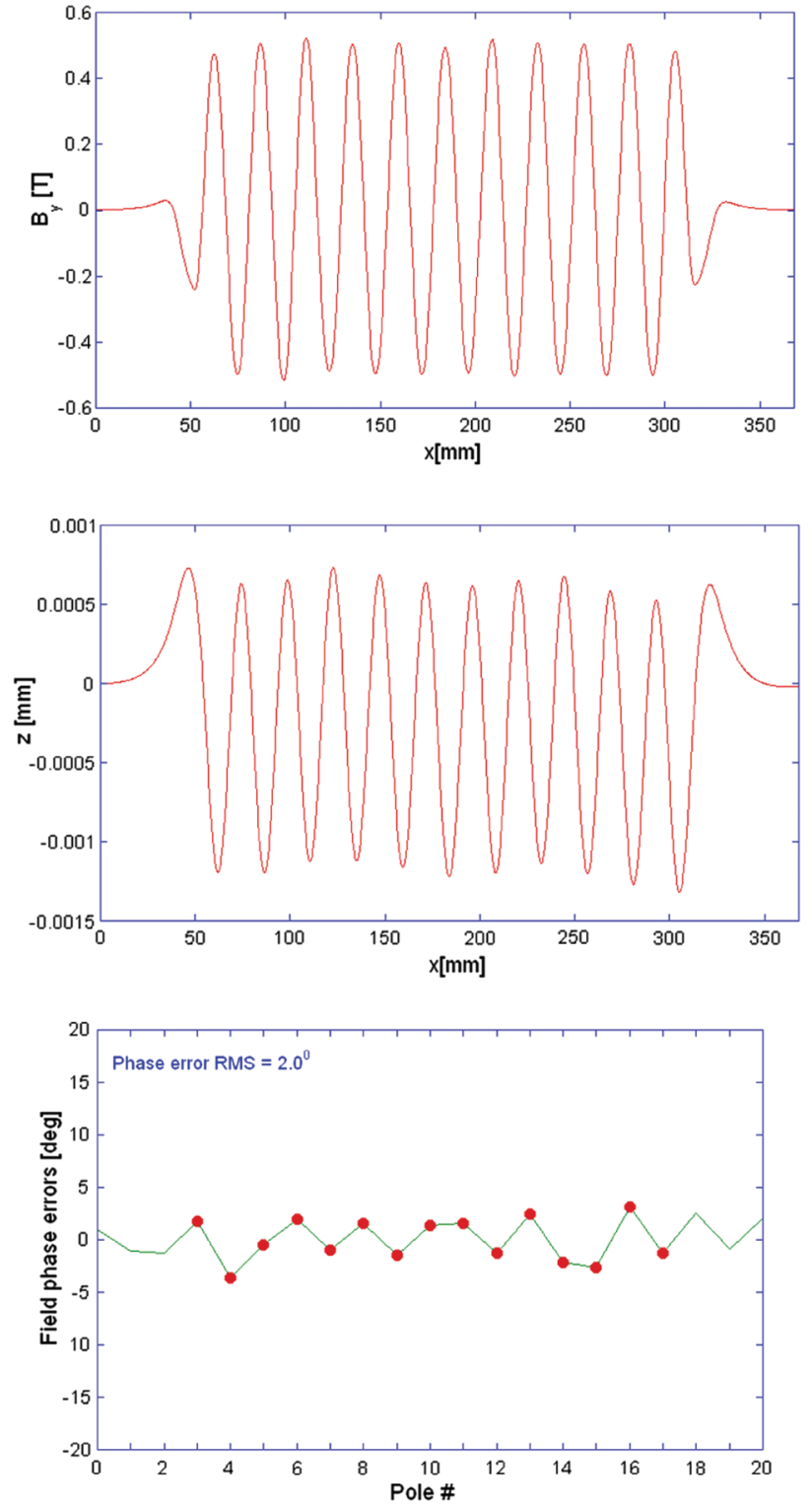

FIG. 13. (Color) Top plot-Array "1" measured magnetic field; middle and bottom plots are beam trajectory and phase errors calculated for doubled field. rms phase errors $\sim 2.0^{\circ}$. 

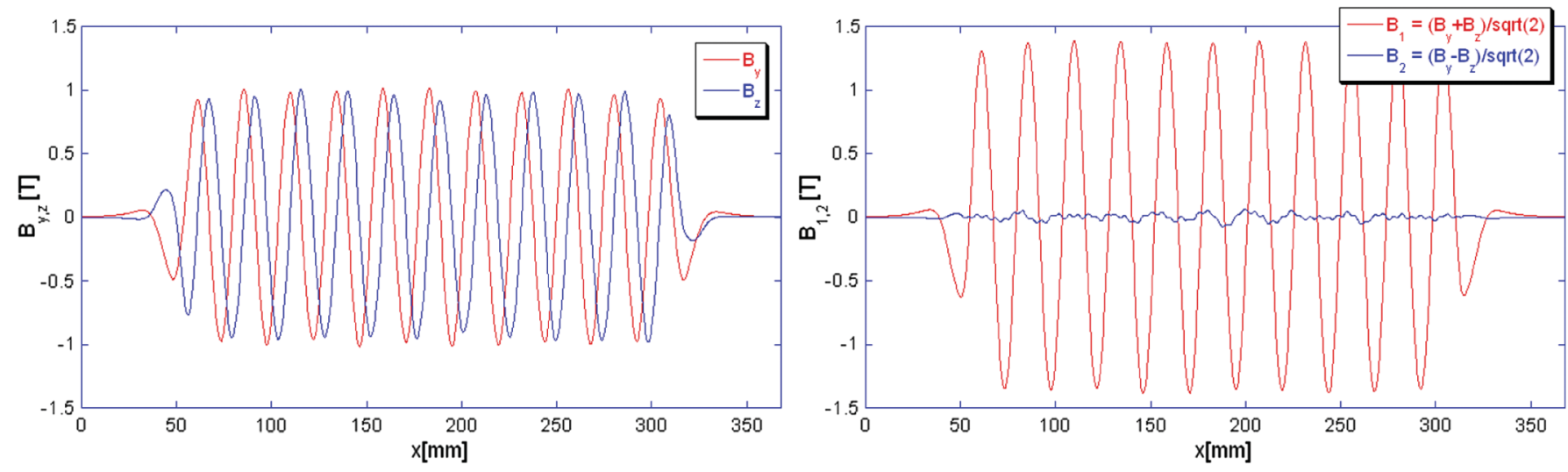

FIG. 14. (Color) Prototype field obtained as a combination of the individual array fields. The left plot shows vertical and horizontal field components for magnet arrays in helical mode configuration. The right plot gives two orthogonal field components for magnetic arrays in planar mode position. For latter rms phase errors $\sim 2.2^{\circ}$.

Cornell ERL "Coherence" mode operation. Results are plotted on Fig. 15. The upper plot shows the spectrum of circular polarized photon flux corresponding to a helical field. There is only one peak at $\sim 1650 \mathrm{eV}$ photon energy. The absence of the other peaks confirms the magnetic field quality. The bottom plot gives the spectrum for linear $\mathrm{x}$-ray
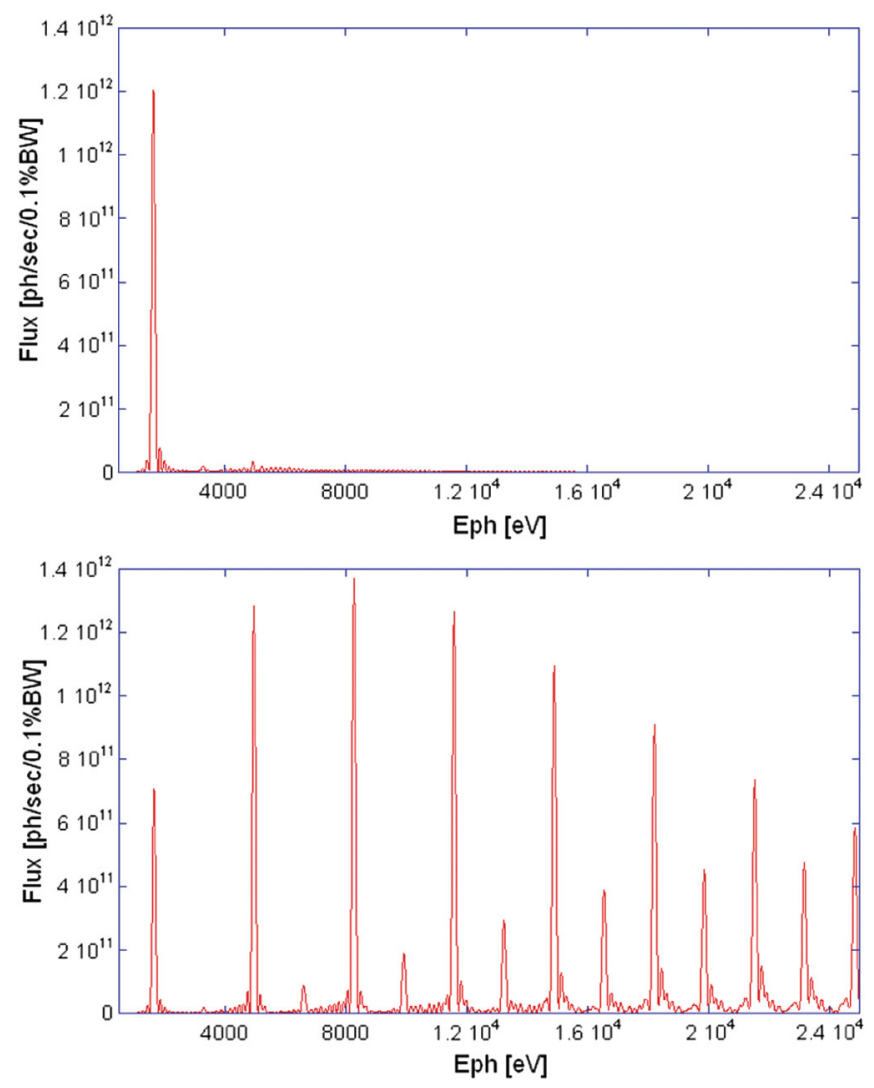

FIG. 15. (Color) Spectrum of the photon flux calculated for the composite field distributions corresponding to the helical (upper) and planar (bottom) mode of operation. polarization corresponding to a planar field distribution. The lowest peak at $\sim 1650 \mathrm{eV}$ matches the first undulator harmonic. Other peaks correspond to higher order odd and even harmonics. Even harmonics appeared because of the finite slit size. The well-defined narrow peaks up to 15 th order indicate small phase errors in the field distribution and confirm the field quality.

\section{NEXT STEPS}

Prior to the full scale Delta undulator design and construction, the following steps will be taken.

In order to verify magnetic field properties inside the $5 \mathrm{~mm}$ diameter undulator gap we will build a special magnetic field measurement setup. In this setup a small size Hall probe will be inserted in the bore and moved along the beam axis. This project is now under way.

To provide the smooth flow of the beam image current between $24 \mathrm{~mm}$ ( 1 in) diameter ERL beam pipe and $5 \mathrm{~mm}$ diameter undulator bore, we have to design special transition pieces. They will be carefully examined for the beam impedance and beam induced power dissipation.

In Ref. [18] we measured demagnetization of the $\mathrm{NdFeB}$ permanent magnets induced by high energy elector radiation. The obtained data allowed us to roughly estimate ID demagnetization rate caused by the beam losses expected at ERL, see [19]. A more detailed analysis will be done after finalizing of ERL optics and beam halo collimation scheme and will be presented in a subsequent paper.

Because we are going to use the Delta undulators for invacuum operation, we will develop and test vacuum cleaning techniques suitable for all undulator components. Traditional in-vacuum high temperature baking at $140^{\circ} \mathrm{C}$ can be applied to all components except magnet arrays. Magnetic arrays can be stabilized against demagnetization at $120^{\circ} \mathrm{C}$ by attaching ferromagnetic plates as described in Ref. [20]. We also will exam the vacuum properties of the 
joints between PM blocks and holders where we used $\mathrm{Sn} / \mathrm{Pb}$ alloy for soldering.

In the Delta undulator prototype, magnet arrays will be moved manually using large screws. For the real undulator we will design mechanisms based on ball screws driving with stepping motors.

In the course of the full scale undulator design study, the transverse and longitudinal beam impedance will be investigated with 3D simulation codes MAFIA [21]. There will also be considered the effect of the bore roughness. The residual gas pressure distribution will be simulated with code MOLFLOW [16].

Coherent synchrotron radiation (CSR) for a wavelength larger than the bunch length is shielded by the narrow beam pipe (undulator bore), but because of the slits between magnet arrays the effective beam pipe of the Delta undulator might be quite large. We will therefore investigate intensity of CSR radiation and possibilities to suppress it.

\section{CONCLUSION}

We have developed, built, and tested a Delta-type undulator prototype which exploits properties of an ERL-type beam. In the design we used nontraditional approaches which led to a number of advantages of the prototype over conventional undulator magnets. Among them are stronger magnetic field, very flexible x-ray polarization control, and compactness. The stronger magnetic field translates to the higher photon flux.

Experience obtained in the course of the work on the prototype will help us build the full scale device.

It should be noted that the Delta-type undulator can also benefit free-electron laser facilities with beam parameters similar to those of ERLs.

\section{ACKNOWLEDGMENTS}

I would like to thank David Rice, Sol Gruner, Donald Bilderback, and Maury Tigner for support of the described activity. My special thanks to Georg Hoffstaetter, Yulin Li, and Kenneth Finkelstein for useful discussions and help. This work was supported by NSF Grant No. DMR
0225180.

[1] Roger Carr, Nucl. Instrum. Methods Phys. Res., Sect. A 306, 391 (1991).

[2] Roger Carr and Heinz-Dieter Nuhn, Rev. Sci. Instrum. 63, 347 (1992).

[3] Roger Carr et al., in Proceedings of 1992 EPAC, Berlin, Germany, 1992, p. 489.

[4] http://sls.web.psi.ch/view.php/beamlines/adress/source.

[5] J. Bahrdt et al., in Proceedings of the 2004 FEL Conference, Trieste, Italy, 2004, pp. 610-613.

[6] Hideo ONUKI, Nucl. Instrum. Methods Phys. Res., Sect. A 246, 94 (1986).

[7] Pavel Vobly, in Proceedings of the Workshop on New Kinds of Positron Sources for Linear Collider, 1997 (SLAC-R-502, CONF-970374), pp. 429-430.

[8] A. Temnykh, CBN 07-13, Cornell, 2007.

[9] A. Temnykh, CBN 06-2, Cornell, 2006, http:// www.lns.cornell.edu/public/CBN/2006.

[10] P. Elleaume et al., Nucl. Instrum. Methods Phys. Res., Sect. A 455, 503 (2000).

[11] Autodesk, Inc., 2008.

[12] ANSYS, Inc.

[13] O. Henry and O. Napoly, Part. Accel. 35, 235 (1991).

[14] Yulin Li, ADC In-Vacuum Undulator Magnet Material Outgassing Test Report, 2008.

[15] John F. O'Hanlon, A User's Guide to Vacuum Technology (Wiley-Interscience, New York, 1989), pp. 33-37, 2nd ed.

[16] R. Kersevan et al., in Proceedings of the 10th European Particle Accelerator Conference, Edinburgh, Scotland, 2006 (EPS-AG, Edinburgh, Scotland, 2006).

[17] T. Tanaka and H. Kitamura, J. Synchrotron Radiat. 8, 1221 (2001).

[18] A. Temnykh, Nucl. Instrum. Methods Phys. Res., Sect. A 587, 13 (2008).

[19] A. Temnykh et al., in Proceedings of the 11th European Particle Accelerator Conference, Genoa, 2008 (EPS-AG, Genoa, Italy, 2008), pp. 214-216.

[20] A. Temnykh, CBN 07-4, Cornell, 2007.

[21] MAFIA, the general purpose electromagnetic code produced by THD-DESY-KFA Collaboration, T. Weiland, 6100 Darmstadt, Germany. 